\title{
INSTRUMENTATION \& TECHNIQUES \\ A device for measuring patterns of locomotor behavior in the earthworm
}

\author{
F. E. McMANUS and EVERETT J. WYERS \\ State University of New York at Stony Brook, Stony Brook, New York 11794
}

\begin{abstract}
A simple device capable of eliciting and detecting earthworm locomotor activity is described and its use outlined. Movement is recorded as an animal crawls in a spoked, revolving wheel. The wheel permits peristaltic crawling, yet restricts the type and timing of other movements. As a result, records of activity differentiate the rate and patterning of peristaltic crawling from changes in bodily position.
\end{abstract}

A persistent problem in the experimental analysis of the behavior of soft-bodied invertebrates has been obtaining objective real-time measurements of activity. Earthworms readily alter their bodily position; consequently, stabilimeters, tilt cages, and photoelectric recording devices seem useful. Unfortunately, earthworms are so slow moving and flexible that minor changes in bodily position produce considerable variability in recorded interresponse intervals unless some type of restriction is enforced. Cinematographic recording allows accurate evaluation of an animal's responses without such restrictions (Collier, 1938; Gray \& Lissmann, 1938), but the method is difficult to use for real-time contingency control, and analysis for regularities is complex and time consuming. A device capable of eliciting response upon which contingencies can be based, at short, regular interresponse intervals, is required.

This note describes a simple device capable of restricting, detecting, and recording earthworm locomotor responses. The device was developed to measure different locomotor patterns after surgical intervention.

The unit consists of a revolving wheel and phototransistor activity recorder. The phototransistor recorder registers the turning of the wheel rather than, directly, the movements of the animal. Patterns of movement are indicated by recording every $30 \mathrm{deg}$ of wheel revolution, and each spoke triggers the phototransistor once. A two-pen cumulative recorder records individual phototransistor counts (after amplification) and the total. This recording system provides a pattern of locomotive behavior over time, as well as total activity.

Earthworms crawl in the wheel readily and persistently. Behavior differences between animals

The authors would like to thank Alexander Castaldi for constructing the wheel and James J. Ashberger for designing and constructing the electronic circuits used. are analogous to those found for other species in a revolving wheel (see Finger, 1972). The use of the phototransistor output to control stimulation of the animal, contingent upon its current performance, is optional.

Direct observation of earthworm activity in the wheel suggests that, during peristaltic crawling, roughly one count is made at each extension of the head, or at each extension of the tail if the animal is backing. The crawling surface of the wheel is " $V$ " shaped, which allows worms of various sizes to have approximately equal lateral contact when turning the wheel. Lateral contact can reduce random changes in bodily position. A restricted crawling space makes changing bodily position, such as turning around, slower than normal.

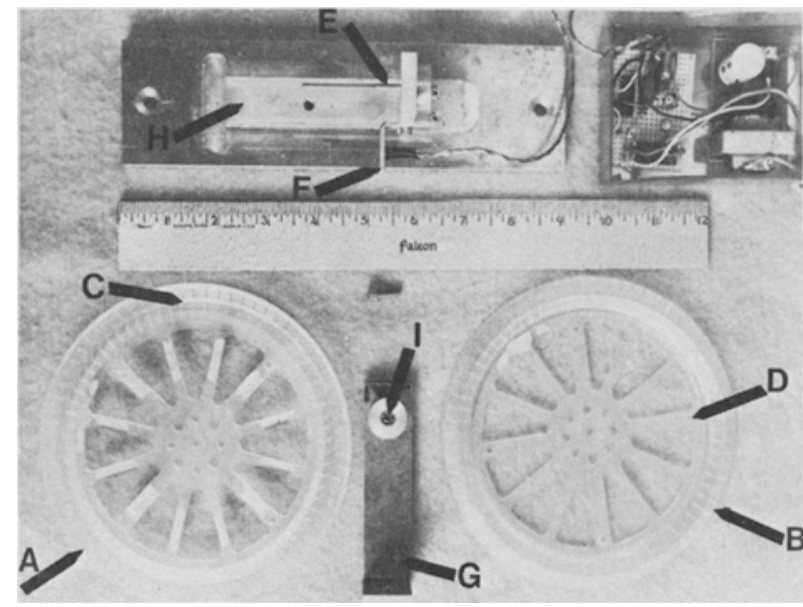

Figure 1. The revolving wheel disassembled and ready for insertion of a worm. (A and B) Two identical halves of the wheel; (C) V-shaped depression in the wheel's rim; (D) one of four symmetrical holes drilled in enlarged spokes; (E) plastic bushing on removable axle; (F) adjustable holder for the phototransistor; (G) removable aluminum upright; $(H)$ groove cut in the aluminum base; (I) stainless steel ball bearing mounts. 
Thus, when an animal is changing position rather than crawling, the recorded pattern of activity is intermittent and widely spaced compared to that of peristaltic crawling. (This system does not differentiate between such changes of position and very slow crawling. If this distinction is required, direct observation is necessary.)

\section{UNIT CONSTRUCTION}

In order to permit easy insertion of an animal, the wheel is constructed in two identical halves. Each half is machined from a Plexiglas block (Figures $1 \mathrm{a}$ and $1 \mathrm{~b}$ ). Using a wooden spatula, an earthworm is readily draped into the open $\mathrm{V}$-shaped depression formed by the rim when one half of the wheel is on a surface (Figure 1c); then, the other half of the wheel can be placed on top, the spokes aligned, and the two halves fastened together. Insertion of an animal in the wheel takes an average of $4.2 \mathrm{~min}(\mathrm{SD}=.6 \mathrm{~min}$ for $\mathrm{N}=20$ ) from removing the animal from its container until the start of recording. Removing the animal from the wheel requires an average of $2.0 \mathrm{~min}(\mathrm{SD}=.4 \mathrm{~min}$ for $\mathrm{N}=20$ ).

The two halves of the wheel are fastened together with four nylon screws. Four symmetrical holes are drilled near the rim of the wheel in four enlarged symmetrical spokes (Figure 1). Pairs of screws are inserted together. Each pair is inserted from the opposite side of the wheel to maintain lateral balance (Figure 2). Then a removable axle (Figure 2) is inserted into the hub of the wheel, until a plastic bushing on the axle

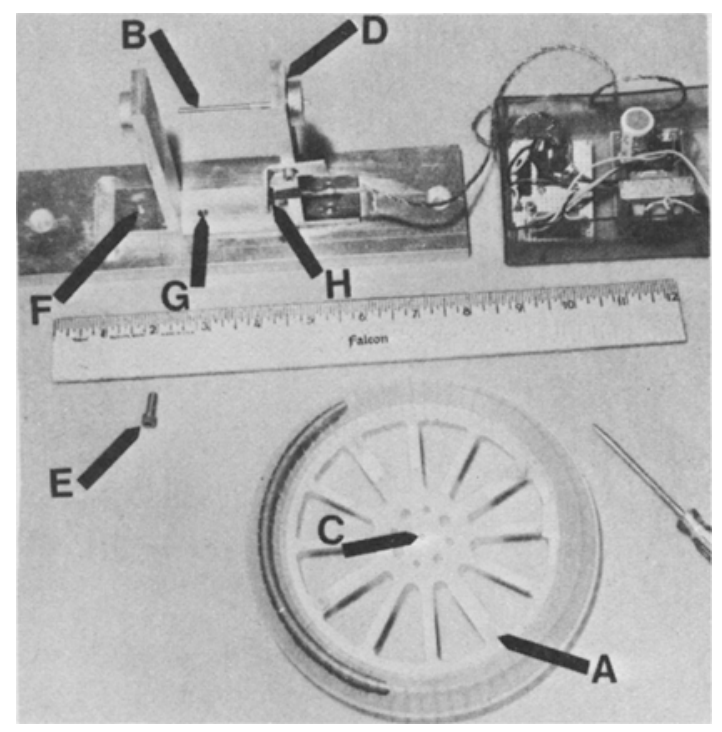

Figure 2. Wheel with worm in place and nylon screws tightened. (A) One of four nylon screws alternated in direction of insertion; (B) removable stainless steel axle; (C) hub of wheel; (D) stationary aluminum upright; (E) thumbscrew; (F) hole in base of removable upright; (G) threaded hole in aluminum base; $(\mathrm{H})$ phototransistor.

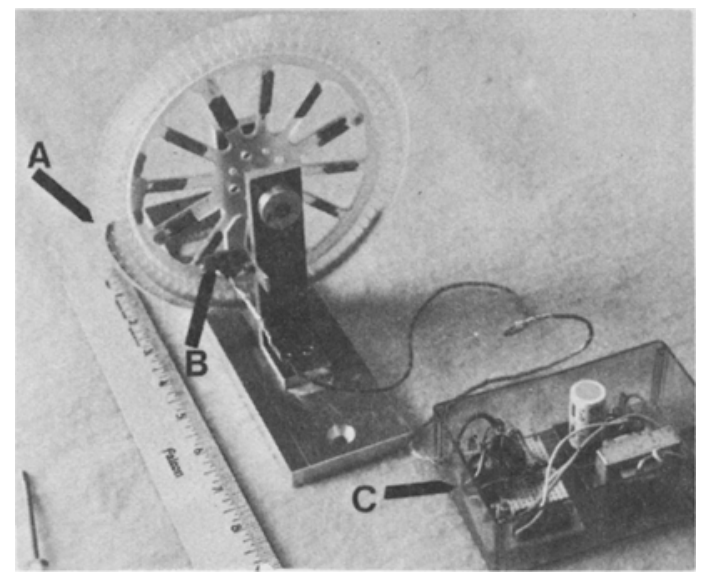

Figure 3. Earthworm and revolving wheel in recording position (cumulative recorder not shown). (A) Head of animal; (B) phototransistor in position to bounce light off a silverpainted spoke; (C) phototransistor power supply and amplifier with long leads.

is encountered (Figure 1). This axle is inserted into the stationary upright upon which the phototransistor is mounted (Figure 2). In this position the wheel is stable and the bushing acts to place the wheel a fixed distance from the phototransistor. The removable upright may then be inserted into a groove cut in the aluminum base (Figure 1) and moved laterally until the upright engages and supports the free end of the axle shown in Figure 2. The removable upright's correct position is determined by manual insertion of a thumbscrew through a hole in the upright's base, and into a threaded hole in the aluminum base (Figure 2).

The assembled wheel and axle weighs $81.6 \mathrm{~g}$. The inside radius of the lowest point of the diamond-shaped crawling space is $7.56 \mathrm{~cm}$. The diamond is $1.06 \mathrm{~cm}$ in diagonal height and $1.70 \mathrm{~cm}$ in width when the wheel is upright. The length of the crawling surface (circumference) is $47.5 \mathrm{~cm}$. Two steps were taken to reduce the resistance an animal encounters in turning the wheel. First, the ends of the axle turn in ball bearing mounts (Figure 1). (These bearings are nonshielded, washed, stainless steel ball, Stock Number 102, from Allied Devices Corporation, Baldwin, New York). Second, spokes are used to connect the rim and hub of each half of the wheel. Spoke thickness is $.15 \mathrm{~cm}$. Removing excess plastic reduces the mass an animal must set in motion. A torque of 3.73 dyne-cm is required to turn the wheel (a .5-g difference in mass between the sides of the wheel at the usual position of the animal's head; see Figure 3). In turning, each of the 12 silver-painted spokes reflects the light emitted by the phototransistor (a Fairchild FPA 105). The circuit diagram for the power supply and amplifier of the phototransistor (Figure 3 ) is available from the authors. Activation of the phototransistor occurs when a moving spoke ceases to reflect emitted light. This signals one 

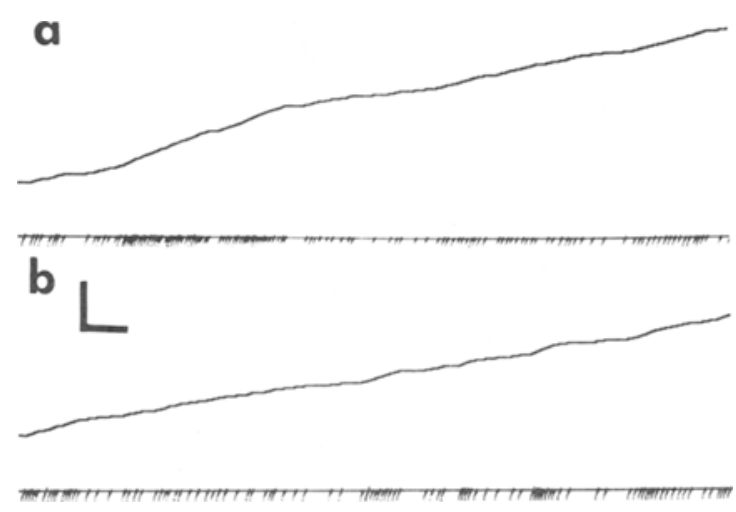

Figure 4. Two 30-min records of earthworm activity in the wheel. (a) Animal weighing $4.19 \mathrm{~g}$; (b) animal weighing $3.90 \mathrm{~g}$. The top line in each figure is the cumulative record; the bottom line is a record of individual spokes counted. Frequent, regularinterval pen deflections indicate steady crawling; isolated, irregular-interval pen deflections suggest changes in position or slow movements on the part of the animal. Vertical scale equals 40 spoke counts; horizontal scale equals $2 \mathrm{~min}$.

count to the cumulative recorder (Ralph Gerbrands Company, Model C-3, not shown).

Each of the V-shaped depressions in the rim of the wheel must be washed between trials. Air holes are drilled at regular intervals around the rim to help retain moisture after washing.

\section{DISCUSSION}

In the wheel, earthworms crawl readily at speeds within the range of $14-30 \mathrm{~cm} / \mathrm{min}$, which is consistent with the peristaltic crawling noted by Gray and
Lissmann (1938), who used cinematographic methods. Records of wheel activity show a typical cumulative record with a relatively constant rate of response. Two .5-h records, produced by different earthworms (both Lumbricus terrestris), are reproduced in Figure 4. These records permit consideration of the rate and patterning of activity separately from total activity. We have found that the average speed in blocks of $1 \mathrm{~min}$ is a useful measure. The longest recording interval has been $1 \mathrm{~h}$, with a $17^{\circ} \mathrm{C}$ temperature and 25 -W red light illumination.

Earthworms are physically able to maintain a steady crawling rate for an entire hour. For example, one animal, weighing $4.54 \mathrm{~g}$, crawled at an average speed of $17 \mathrm{~cm} / \mathrm{min}$ (SD $=10 \mathrm{~cm} / \mathrm{min}$, measured for each of the 151 -min blocks) for the first $15 \mathrm{~min} ; 25 \mathrm{~cm} / \mathrm{min}$ $(\mathrm{SD}=6)$ for the second $15 \mathrm{~min} ; 23 \mathrm{~cm} / \mathrm{min}(\mathrm{SD}=4)$ for the third $15 \mathrm{~min}$; and $25 \mathrm{~cm} / \mathrm{min}(\mathrm{SD}=4)$ for the last $15 \mathrm{~min}$. The fastest speed for the 50 animals tested was $88 \mathrm{~cm} / \mathrm{min}$ for the first minute after insertion in the wheel.

\section{REFERENCES}

Collier, H. O. J. The immobilization of locomotory movements in the earthworm, Lumbricus terrestris. Journal of Experimental Biology, 1938, 15, 339-357.

FINGER, F. W. Measuring behavioral activity. In R. D. Myers (Ed.), Methods in Psychobiology (Vol. 2). London: Academic Press, 1972.

Gray, J., \& LissmanN, H. W. Studies in animal locomotion: VII. Locomotory reflexes in the earthworm. Joumal of Experimental Biology, 1938, 15, 506-517.

(Received for publication November 10, 1977; revision accepted January 5,1978 .) 